\title{
El Padrón Municipal de Beneficencia y la geografía de la pobreza oficial sevillana c. 1905.
}

\author{
The Charity Town Census and scaning of the official poverty in \\ Seville (Spain) c. 1905.
}

JUAN VILLA LÓPEZ

\section{RESUMEN}

Este trabajo se sitúa entre los años 1904-1906 en Sevilla (España). Se estudia el intento en aquellos años de aligerar las grandes listas de acogidos al Padrón Municipal de Beneficiencia por el coste médico y farmaceútico. El Padrón Municipal

se denominaba, a principios del siglo $X X$, al censo en el que estaban inscritas las personas susceptibles de recibir asistencia sanitaria por parte del ayuntamiento. Al conocer las condiciones para la inscripción, prácticamente todos los asalariados

sevillanos ( $y$ españoles) podrían formar parte del Padrón. La causa principal es evidente: la miseria del jornal del obrero. Además, se analiza la distribución urbana de los acogidos y su concentración en zonas determinadas que configuran una verdadera geografía urbana de

la pobreza con una enorme carga política y social.

PALABRAS CLAVES:

Padrón Municipal de Beneficiencia, pobreza, vivienda, Sevillia, 1904

\section{ABSTRACT}

This work is located in the years 1904-1906 in the city of Seville (Spain). It is studied how the town council tried, in these years, to diminish the large list of people registered in what wasis called Padrón Municipal de Beneficiencia (Charity Town Census in English) because it was a great cost, medical and pharmaceutical, for the town public funds, in that époque of shortage and stagnation. The Charity Town Census was defined, at the beginning of last century, as the census in which were registered people susceptible of receive sanitary assistance from the Town Council. Given the conditions for the registration, almost every wageworker in Seville (and Spain), could take part of the Charity Census. The main reason for this situation was obviously the paltry salary of the workers. Furthermore, it is analysed the city distribution of people belonging to the Census and their concentration in particular areas of the city, shaping a true urban geography of poverty, with an enormus political and social content.

KEYWORDS:

Charity Town Census, poverty, housing, Sevilla, Spain, 1904 


\section{EL PADRÓN MUNICIPAL}

El presente trabajo contiene dos partes muy diferenciadas. En la primera se analizan diversos aspectos legales seguidos del estudio de los cambios que pretende el Ayuntamiento a través de una punta de lanza que era el edil Barón y Martínez de Agulló, conde de Colombí y futuro alcalde y las propuestas e intervenciones de los Farmacéuticos y Médicos municipales. Se piden medidas restrictivas y los galenos reciben diversas acusaciones de las que se defienden y defienden, los únicos, a los enfermos inscritos en el Padrón Municipal de Beneficencia. La segunda plasma sobre el papel la realidad geográfica y palpable de dichos inscritos teniendo en cuenta que al final, enfermedad, pobreza y geografía urbana están intimamente ligadas. Ambas partes se complementan si tenemos en cuenta lo que opinan los propios médicos cuando indican a los políticos municipales que hay Padrón porque hay pobres que necesariamente se acogen a él y si hay pobres se deberá atender a todos, y bien.

Mal año, 1904, escogieron los ediles sevillanos para revisar y adelgazar el número de acogidos al Padrón Municipal de Beneficencia'. Que el tema les preocupaba nos lo demuestra la interminable serie de intervenciones a favor de su revisión a la baja y las nuevas propuestas para el nuevo fichero que, sin duda alguna, según ellos, daría una nueva realidad asistencial.

Las Actas Capitulares del Ayuntamiento de Sevilla mostraban la preocupación económica y de imagen de las autoridades ante un Padrón que les era adverso por su propia naturaleza y por la estructura salarial de los trabajadores sevillanos. Arenas nos informa de que ganando un trabajador entre tres y cuatro pesetas diarias y entre tres y cuatro miembros de familia ya tenía derecho a inscribirse en el Padrón ${ }^{2}$, con lo cual el número de acogidos era realmente impresionante porque ese salario estaba fuera del alcance de la inmensa mayoría de los obreros. Un médico de la Beneficencia venía a ganar algo más de seis con cincuenta pesetas diarias.

La Ley de Sanidad de 28 de junio de 1855 no habla en ningún momento de los Padrones propiamente dichos y desarrolla en su articulado la forma-

Archivo Municipal de Sevilla. Expedientes Especiales. Legajo 1547. Esta fuente primaria ocupa una caja con dos legajos correspondientes a dos épocas diferentes. Nosotros hemos trabajado con la segunda época referida a 1903-1.905 y a él nos referimos siempre. Los datos que se comentarán, provienen todos de dicho Legajo 1547. Más adelante, en la página 3 se define y clarifica lo que era el Padrón y lo que suponía para el municipio.

${ }^{2}$ Arenas Posadas, C., La Sevilla inerme, Écija, 1.992, pág. 93. En Sevilla y el Estado, del mismo autor, pág 70, se nos informa de que el 96 '51\% de la contribución en Sevilla, lo formaban las Cédulas Personales que iban desde las 750 pts anuales a las negativas de los jornaleros, o sea, desde la novena a la undécima clase en el año 1.890 y el 96 '51\% en 1.907 . 
ción de Juntas Provinciales y Municipales de Sanidad; se ocupa de los facultativos médicos en los artículos 64 a 80 y de los farmacéuticos del 81 al 89 , pero no hay articulado alguno sobre los que podían ser considerados como pobres y beneficiarse de las leyes, salvo que sean pobres. No se regula, pues, este concepto, que debía ser muy general necesariamente teniendo en cuenta los salarios que percibía un jornalero, por ejemplo. En 1848-60 ganaba un bracero según Bernal, 4,2 reales ${ }^{3}$. En 1910, según el mismo Bernal, el jornal medio en Sevilla era de 2'10 pts. los hombres; de1'01 las mujeres y de 1'03 los niños ${ }^{4}$.

Dado que los Reglamentos y Reales Órdenes debían ser poco efectivos, Silvela, Ministro de la Gobernación a la sazón, propone a la firma de la Reina Regente del reino, un reglamento con fecha de 14 de junio de 1.891 en el que ya se contempla y regula el concepto de pobreza en los siguientes términos y en su artículo 3: «Serán considerados como vecinos pobres para los efectos del reglamento:

1. - Los que no contribuyan directamente con cantidad alguna al Erario ni sean incluidos en los repartos para cubrir los gastos provinciales ni municipales.

2. . Los que vivan de un jornal o salario eventual.

3. Los que disfruten de un sueldo o pensión menor que los de un bracero en la localidad respectiva y cuenten con aquel solo recurso.

4. - Los huérfanos pobres y expósitos que lacten y se críen por cuenta de la Beneficencia pública en las respectivas jurisdicciones».

Pasada la Restauración, pasada la Dictadura de Primo de Rivera, al final de la Guerra Civil, el que luego fuera Ministro de Justicia franquista, Antonio Iturmendi Bañales, Director General de Administración Local, envía una Circular con fecha 4 de diciembre de 1939, Año de la Victoria, dando normas para la renovación del Padrón de familias pobres de la Beneficencia Municipal (se conoce que esto de la pobreza también se heredaba) y para la calificación de pobreza recurre «bien a los requisitos establecidos en el artículo tercero del Real Decreto de 14 de junio de 1891, ya a otras normas...» por lo que el reglamento de Silvela estaba de pleno vigente.

Como se puede comprobar con todo lo legislado, lo que se crea es una red completa de carácter nacional para la atención primera de los pobres cuya hospitalización no fuera necesaria, bien es verdad que al Hospital Provincial sólo iban los pobres. Esta red es de primordial importancia, pero tam-

\footnotetext{
${ }^{3}$ Bernal, A. M. , "Señoritos y jornaleros : la lucha por la tierra», en Historia de Andalucía, vol. VII, Barcelona 1.981, pág. 292.

${ }^{4}$ BERNAL, A. M., «El rebaño hambriento en la tierra feraz", en Historia de Andalucía, vol. VIII, Barcelona, 1.981 , pág. 86.
} 
bién una carga para los Ayuntamientos que intentan por todos los medios sacudirse al pobre. Pero se instalaba la normalidad de una red asistencial general y barata, máxime en las ciudades y pueblos andaluces de gran población, si bien dejaba fuera cortijadas, aldeas, núcleos muy pequeños, etc. En 1963 se aprueba la Ley General de Bases de la Seguridad Social que cambia el sistema asistencial.

El Padrón Municipal de Beneficencia estudiado respondía al Real Decreto de 1891 con el objetivo evidente de dar cobertura médica y farmacéutica a la mayoría de los habitantes de una ciudad o pueblo (obsérvese cómo los españoles estaban indefensos ante la enfermedad y su curación) que no podían pagar la medicina particular o algún tipo de iguala por la exigüidad del jornal. Se trataba, pues, en primer lugar, de una asistencia puramente benéfica que venía a asumir, de manera un poco vergonzante, antiguas prerrogativas de la caridad privada que, no obstante, seguía funcionando en otros aspectos como el de la asistencia hospitalaria, asilo y acogida y a través, en nuestro caso, de la Asociación Sevillana de Caridad.

En segundo lugar, el Padrón era una losa auténtica y continuada para la economía de los municipios y sus presupuestos. Por eso, los intentos de enmagrecer esta asistencia son normales en este sentido. Nadie quería ver, naturalmente, las causas de este elevadísimo número de censados.

En tercer lugar era una extraordinaria radiografía, sorprendente por su exactitud, de la pobreza de la clase trabajadora incapaz de atender sus mínimas necesidades, de tal modo que los obreros eran considerados como pobres reales, pero todavía en una escala superior a otros aún más necesitados que clependían de otro tipo de caridad o beneficencia. En ambos casos, eran hombres que no tenían ni una opción ante la vida. Su historia estaba disociada de la historia política y nos los vamos a encontrar unidos siempre a la protesta social en todas sus manifestaciones. ${ }^{5}$

Este yugo al que los ayuntamientos estaban forzosamente uncidos daba lugar a diversos abusos e intervenciones de la picaresca, que se convertían así, en excusas para revisar, a la baja naturalmente, los afiliados al Padrón. Pero además había otro interés, un tanto peregrino y extraño que era el de la imagen pública, por una parte y la autocomplacencia por otra dentro de una ceguera general ante los aspectos sociales. En este sentido, el edil sevillano Barón, conde de Colombí y político de primera magnitud, plantea las cosas de manera bien clara: «... y esto (el número de pobres y asistencias médicas) es imposible por ser Sevilla centro industrial y comercial, a la altura de las capitales más florecientes, no ya de España sino de Europa» ${ }^{6}$. Ante este tipo de

${ }^{5}$ González Fernández, A., Lucha obrera en Sevilla. Conflictividad social 1900-1907, Barcelona, 1988, págs. 165-177.

${ }^{6}$ Archivo Municipal de Sevilla. Padrones Benéficos, 1904, Legajo 1547. 
declaraciones no sabemos si asombrarnos más de la manipulación egocéntrica, la ignorancia falaz o era la visión propia de la oligarquía sevillana.

Es el antedicho Barón el que en 1904 levanta la veda ante el problema del Padrón que va a ocupar el funesto 1905, año de fortísima crisis. Hace notar que el Padrón vigente responde al formado en el año $1896^{7}$ y que en estos años podía haber cambiado la definición de pobreza. Algo realmente imposible sin que cambien las estructuras salariales, que no habían cambiado; alega además que se cometían abusos y que se debería confeccionar un nuevo padrón, esta vez realizado por la Asociación Sevillana de Caridad que contaba con "un servicio de investigación de los verdaderos pobres» según las nuevas maneras que se querían imponer. Confiaba, pues, Barón en una institución privada, que también recibía fondos públicos, más que en los servicios del Ayuntamiento al que pertenecía. La Asociación se convertía en el factótum de los designios del propio edil. Se convertía en el Gran Elector de la miseria.

Pero no iban por ahí todos los tiros porque el Real Decreto de 1.891 estaba vigente y era intocable, pero no otras realidades, principalmente las de valor económico. El valor económico de las prestaciones se podía rebajar de dos maneras: se eliminaban pobres o se suprimían o se ahorraba en los gastos que las enfermedades de éstos provocaban. Dicho de manera más clara, gastos médicos o gastos farmacéuticos, porque lo que era imposible eliminar era el número de pobres que había y los que se avecinaban con el tremendo $1905^{8}$, salvo que se actuara draconianamente sobre el censo benéfico ignorando y forzando la ley. Pero será sobre los gastos susodichos sobre los que se va a librar una lucha entre los ediles y los causantes del gasto, que no son ahora los enfermos, que también, sino los galenos y boticarios municipales, lucha desigual porque éstos, ante ellos mismos y hasta hoy día, casi un siglo después, son capaces de salir vencedores y garantes de la sanidad que hoy llamaríamos pública aplicada muchos años antes a las sociedades europeas de las conquistas sociales.

El valor que para el pobre tienen los médicos es inimaginable hoy y está fuera de toda duda, pero contra ellos como Corporación de la Beneficencia, se dirigirán los esfuerzos ahorrativos de la corporación hispalense. $Y$ en dos direcciones: las visitas médicas que se consideran excesivas y las recetas médicas. Aquí entra un nuevo factor no despreciable para los ediles: las recetas y con ellas los farmacéuticos y las Farmacias Municipales. Vemos ya a médicos y farmacéuticos en el punto de

\footnotetext{
${ }^{7}$ Actas Capitulares del Ayuntamiento de Sevilla. Libro VI, sesión del 7 - X-1.904.

${ }^{8}$ La crisis agrícola afectó a todo el país de diversa manera. Sus efectos en Andalucía y Extremadura fueron tremendos por la especial estructura de la posesión agraria y la temporalidad del trabajo. La causa a la que todos se refieren fue la sequía. Para Hobsbawm en Rebeldes primitivos, fue la última verdadera hambre a excepción de algunos episodios que siguieron a la guerra civil.
} 
mira de los gobernantes municipales. Pero como el R. D. estaba plenamente vigente, como hemos dicho, las opciones de ahorro estaban claras, con algunas variantes que comentaremos, pero que eran menores por su representación social y política.

Los galenos eran conminados desde el poder municipal a que «dentro del cumplimiento de sus deberes profesionales, se inspirasen al redactar sus recetas, en la más extricta (sic) economía", lo que significaba una real dilapidación, hasta el momento, de los dineros públicos puesto que si se les llamaba la atención era porque se consideraba recetaban las medicinas más caras. No es el momento de revisar los petitorios farmacéuticos ${ }^{9}$, pero, desde luego, éstos eran realmente baratos y económicos y en todo el escrito se nota una cierta contención y cuidado hacia el Cuerpo de la Beneficencia Municipal que, claro está, no eran obreros ni menesterosos. Pero en el consejo ya estaba la acusación. Y como nota general de la irresponsabilidad municipal cabe destacar que en ningún momento y ninguno de los que intervienen en esta lucha por el adelgazamiento de la pobreza oficial municipal, ninguno de los intervinientes, salvo los médicos, se pregunta por la causa de la existencia de un Padrón, según ellos, tan gordo y lleno de salud, por las causas de esta pobreza que era algo más, miseria, pura y clara.

Barón y Martínez de Agulló encuentra dos elementos claves para pedir un nuevo Padrón: Dice que entre las 61.820 asistencias de 1896 y las 86.000 de 1904 hay un aumento de veintiuna mil y esto no puede ser, Sevilla no puede tener tantos pobres según su lógica manera de pensar; en segundo lugar acusa a los médicos de prodigalidad. Pero esta acusación venía a aumentai el malestar que debían tener todas las autoridades con la actividad crítica de los médicos, ya municipales, ya particulares, algunos de los cuales eran verdaderos látigos para la autoridad municipal y gubernamental y, desdichadamente, siempre con la razón por delante. Desde el benemérito Hauser ${ }^{10}$ hasta Sánchez Pizjuán, los médicos habían denunciado, de manera muchas veces acre, las condiciones de vida de los obreros y pobres, del estado de las zahúrdas que habitaban, la alimentación escasa y pobre que no servía ni para reparar las fuerzas perdidas tras jornadas agotadoras que poco a poco, cuando no en tropel, conducían al sevillano pobre a la muerte. El mismo y batallador Sánchez Pizjuán llamaba "muriendas» ${ }^{11}$ a las viviendas en las que moraban estos sevillanos, término que podría formar parte del léxico quevedesco o valleinclanesco sin desdoro. Otro de los escritores

\footnotetext{
${ }^{9}$ Archivo Municipal de Sevilla. Farmacias Municipales, legajos $410-412$.

${ }^{10}$ HAUSER, Ph., Estudios médicos topográficos, Sevilla, 1982; del mismo, La verdad sobre las aguas potables de Sevilla,Sevilla, 1883 y El pauperismo en Andalucía y singularmente en Sevilla, Madrid, 1884. Importante es también su Madrid bajo el punto de vista médico-social, Madrid, 1902.

$"$ Citado por AREnAS en La Sevilla inerme, pág. 37.
} 
más luchadores, Chaves Nogales ${ }^{12}$, dice «... son las más dolorosas habitaciones de proletarios que conocemos. Estrechas, oscuras, húmedas, ruinosas, carecen de esa uniformidad carcelaria que a las barriadas obreras impone la higiene moderna, y de aquella saludable anarquía de nuestros corrales". Hay que hacer notar que los 'corrales' no eran las viviendas más indignas de Sevilla. ¿Cómo serían las otras? Con estos mimbres sanitarios se fabricaban los enfermos que cuidaban y recetaban los médicos municipales. ¿Servían de mucho estas humildes medicinas? Según los tantos por mil de fallecimientos y la lista de enfermedades del Hospital Central, de bien poco, si es que les daba tiempo de ser internados y reducidos a unas simples tablas estadísticas.

Pero el Conde de Colombí dice algo más y algo más grave e insultante por lo que tiene de desprecio a la inteligencia humana. Dice el Conde que a los pobres se les receta más que a los particulares pudientes. Este racismo económico al revés es cómico si no fuera trágico y este pensamiento del dispendio para pobres debía ser normal para buena parte de la Corporación, porque nadie levanta la voz para protestar y desautorizar tamaño disparate, sobre todo si echamos una ojeada a los petitorios farmacéuticos y constatamos lo elemental de las peticiones.

Barón añade algo reiterativo en la beneficencia y es el préstamo de las cédulas. Otro dato es que los miembros de la Guardia Civil no deberán ser integrados en el Censo porque, como estamento militar, ya cuentan con médicos militares. Ya veremos como esta cuestión se va olvidando en esta lucha económica; igualmente tampoco se concederá este derecho a las trabajadoras, varios miles, de la Fábrica de Tabacos ya que tienen su propio concierto por otra parte. Además, el petitorio farmacéutico deberá ser vigilado y se eliminarán pedidos como el de vino con el que se hacía el vino quìnado que, al fin y al cabo, era en la época algo más que un reconstituyente.

Y dentro de la más estricta moral decimonónica y sentido de la caridad cristiana, anota Colombí desde sus 29 años de caritativa dureza, que la Cédula $^{13}$, "sirva [deberá servir], como acicate o premio para las familias pobres, que vivan honradamente, cumpliendo sus deberes para con Dios y la

\footnotetext{
${ }^{12}$ Chaves Nogales, M., La ciudad, Sevilla 2001, pág. 112. También la 73. Hablando de los corrales los define en la misma obra y página así. «Tienen un amplio patio de relucientes guijos, una fuente en el centro, unas galerías donde los viejos y los niños salen a tomar el sol, y un centenar de salas». Alcalá Venceslada en su Vocabulario andaluz, Jaén, 1998, nos dice que la sala es «Vivienda familiar en los corrales y corralones de vecindad de algunas localidades andaluzas"; de Los corrales, de MORALES PADRón, hay reimpresión de 1981 en Sevilla, es un texto clásico. En definitiva, era una casa de vecindad habitada por pobres con un patio al que daba el sol y era zona comunal. Para Arenas en la citada La Sevilla inerme, pág. 100, es mejor que las denominadas "casa de vecinos", «casas de pisos» 0 «departamentos», generalmente de peores condiciones higiénicas. O sea, dentro de las infraviviendas inhumanas sevillanas había sus grados. En el Censo Oficial de 1900 existen 1,118 corrales en los que se calcula que viviría dos tercios de la población de Sevilla que en 1900 era de 148.315.

${ }^{13}$ Archivo Municipal de Sevilla, Padrones de Beneficencia, 1904, Legajo 1547.
} 
sociedad y los propios del hogar doméstico.... siendo útiles y no perjudiciales en su esfera social a sus conciudadanos de más holgada fortuna... [ y tendrá] las más puras satisfacciones al ver premiada la honradez, la laboriosidad y la moralidad en las costumbres por mano del Excelentísimo Ayuntamiento que no puede prescindir de la justicia en ninguno de sus actos, ni por lo tanto, olvidar la diferencia que existe entre la virtud y el vicio, entre el bien y el mal, entre la laboriosidad y la holgazanería, entre el ahorro prudente y el despilfarro imprevisor..." y un largo etc. etc. y todo por ganar dos miserables pesetas y estar cargado de hijos, vivir en una pocilga y no comer. Para colmo de males lo van a depurar. Este pesadísimo e insufrible sermón santurrón que hunde sus raíces en años muy anteriores, tiene un tono "antiguo régimen» realmente español, más santurrón que moral, nada ético y profundamente católico basado en la jerarquía estricta, en la escala superior/ inferior. No era otra la moral de los chistes de postguerra en los que el señorón o la señorona daban diez céntimos a un pobre y le conminaban a que no se los gastara en vino.

Las aportaciones que vamos a apuntar vienen en cascada y responden a la tala deseada de menesterosos. Pero llama la atención de manera poderosísima que los tres taladores de pobres que nombramos a continuación y que brindan sus ideas para enflaquecer el Padrón, son los tres farmacéuticos y directores de las Farmacias Municipales de Santiago, Calatrava y Trastamara. Así, Luis de la Cuadra, propone que se exceptúe del Padrón a los siguientes posibles perceptores de la beneficencia:

- Los que contraigan enfermedades adquiridas en costosos vicios inmorales pagados espléndidamente ( $¿$ ?) para venir a curarse a costa de los fondos municipales.

- Los que vivan de forma irregular, no ajustada a matrimonio católico.

- Los que tengan antecedentes penales, especialmente los reincidentes.

- Los sexagenarios no imposibilitados para el trabajo y vivan de manera ociosa.

Como es fácilmente comprensible, el número de acogidos a la caridad municipal bajaría vertiginosamente.

Pero otros personajes presentan alternativas y mejoras para la poda; así, de Pablos y Sánchez Castañer añaden algunas ideas más, pero ya se han olvidado de un colectivo tan querido por las fuerzas políticas de la Restauración como es el de la Guardia Civil, aunque persistan en la baja de las cigarreras belicosas socialmente.

Sánchez Castañer, que había sido edil y había estado en la comisión que bajo la presidencia de Cañal Migolla creó la Asociación Sevillana de Caridad, propone entre otras medidas la no asistencia a los portadores de en- 
fermedades venéreas o sifilis. En este sentido, queremos recordar la poca incidencia que encontramos de estas enfermedades en el Hospital Central de Sevilla frente a la gran cantidad de muertes de niños afectados por ellas en las defunciones registradas en la Casa-Cuna, en realidad, a pesar de todas las incidencias, el tanto por ciento de mortalidad por ellas es del $0{ }^{\prime} 5 \%$ de la mortalidad total de la ciudad, que, por otra parte, en el año 1.901 tenía 41 burdeles oficialmente inscritos y con asistencia médica garantizada, aparte la prostitución callejera en las variantes más enrevesadas y siempre clandestina, si bien perfectamente conocida por las autoridades. Esta sifilifobia resultaba realmente obsesiva en los ambientes tan religiosos sevillanos. Éste también se olvida de las cigarreras y de la Guardia Civil pero no de la parquedad en el uso de las recetas por parte de los médicos.

Ante tantos ataques los médicos se defienden con una gallardía y energía extraordinarias y lo hacen a través del Decanato del Cuerpo Médico Municipal en escrito dirigido al Inspector del Cuerpo de la Beneficencia Sanitaria Municipal. Se trata de una hermosa pieza en defensa de sus colegas desde la más estricta categoría profesional. Pero es más importante e interesante la defensa que se hace del enfermo mucho más próxima al Cristo del madero que a otros Cristos. En resumen dice:

- Hay muchos establecimientos a los que atender y por lo tanto el número de recetas y enfermos aumentan.

- No se pueden restringir gastos si antes no se restringe el número de pobres. Mientras existan pobres, se apuntarán con toda seguridad al Padrón. Hay gasto porque hay pobres. Con esta simple aseveración da el Decanato en la diana social de la Restauración que se mostraba incapaz de parar el aumento de necesitados y el desequilibrio social.

- Atienden a 1.200 familias por médico, cuatro veces más de lo que marca la ley y nadie en el Ayuntamiento le ha dicho nada en ningún sentido.

Como las anteriores hay varias justificaciones más que harían sonrojar a más de un concejal. La pieza que comentamos y reproducimos en nuestro Trabajo de Investigación ya citaclo, no tiene absolutamente ningún desperdicio por su misma grandeza y asíi ${ }^{4}$ :

- La Beneficencia Municipal no debe considerarse como una función administrativa más ordenada por la ley, sino como un deber que la sociedad tiene para con el pobre, el enfermo pobre.

- Hay que llamar la atención por la claridad y grandeza del pensamiento médico. 
- La causa de la enfermedad quizá resida en la extremada dureza del trabajo y la falta de recuperación del desgaste de energías por insuficiencia de medios para cubrirlos.

- Las miserables condiciones de las viviendas en las que habitan los censados.

- Los ediles sabían esto perfectamente, mejor que nadie, a excepción de los propios médicos.

- No se puede ahorrar en medicamentos que sirvan para paliar el dolor, a pesar de que en el expediente se pida el uso de medicamentos más baratos, aunque causen molestias al enfermo.

- Son dignas de ser comparadas ambas posturas.

- Eliminar del Padrón a quienes no sean un ejemplo, dicen, no es ni humano ni caritativo.

- Sobre las enfermedades venéreas y sífilis, opinan los médicos que hay que ayudarles y perdonarles si en ello hay pecado, como hizo Jesús.

- Luego se extienden en varias consideraciones como que los jóvenes son arrastrados al vicio, que no se les puede abandonar y que hay que incorporarlos al trabajo y a la economía nacional.

- La ciencia y los hechos nos enseñan que la riqueza de los pueblos y su bienestar se halla en razón directa con la salud de los individuos que la forman.

- Bien sabían los médicos, como los dirigentes políticos que los índices de mortalidad nacionales o sevillanos pertenecían a lo que hoy llamaríamos tercer mundo.

- Conforme a sus obligaciones, dicen, deben curar a los enfermos, por más que recaigan.

- Protestan por las acusaciones presentadas por quienes nada saben de la asistencia a los pobres.

- Esta es una defensa de altura porque en el Consistorio había varios médicos.

Hasta aquí parte del proceso de depuración del Padrón y la defensa de los médicos ante las acusaciones y peticiones de los consistoriales sevillanos. 


\section{II}

En esta segunda parte estudiamos la distribución de los inscritos en el Padrón por parroquias y distritos ubicados en los barrios a los que se refiere el título de este trabajo. Necesariamente el localismo es irremediable, como lo sería un trabajo sobre Barcelona o Bilbao, pero para establecer algún tipo de contraste, véase la situación de los barrios pobres madrileños con cifras de hacinamiento tremendas y de mortalidad escandalosa si las comparamos con barrios ricos, v.g., así, Inclusa, Latina, Embajadores, Peñuelas etc., frente a Centro o Buenavista; la diferencia de mortalidad es de 18,30 puntos. La insalubridad afectaba a más de la mitad del área urbana habitada por obreros y necesitados. Hauser dice que la población de las casas de vecindad de Madrid, se componía en su mayoría «de la clase jornalera, de empleados cesantes, de vendedores ambulantes, de barrenderos y traperos " 15 . También podemos observar las condiciones higiénicas reinantes en una ciudad de estructura social y económica y, sobre todo, de un talante democrático tan diferente, en la obra de Reig ya citada en la nota 22.

En enero de 1905 aparecen una serie de datos con el número real de los inscritos en el Padrón hasta este año es una cuenta muy ajustada y fruto de una petición municipal y lo avala la firma del Secretario de la Corporación

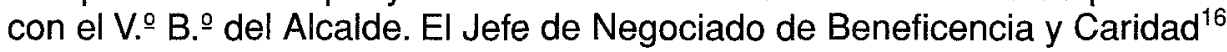
informa de que el número de familias inscritas es de 14.052 que siempre nos dará una cifra total de $\mathbf{5 6 . 2 0 8}$ si multiplicamos por cuatro la unidad familiar y de 70.260 si lo hacemos por cinco. Recordemos que el Censo Oficial de 1900 era de 148.311 y el de 1910 de 158.287 habitantes. Si dividimos el crecimiento censal entre dos, ya que estamos a mitad de la década, tenemos la cifra de 4.988 nuevos censados que sumada a 148.311 nos da un total de 153.299 a mitad del periodo intercensal. Esto supone que en el primer caso de la unidad familiar, sería el $36^{\prime} 66 \%$ del total del Censo si la consideramos de cuatro miembros y en el segundo caso el $45{ }^{\prime} 83 \%$ con cinco miembros por unidad. Estos son los datos sobre el Censo ideal.

Sin embargo, en una nota a mano, sin membrete ni nota distintiva o firma, salvo que está en el mismo legaio, se nos informa de que el número de inscritos en el Padrón es de 64.632 personas, el $42 ' 16 \%$ del Censo medio de crecimiento censal. Se añade que hay 13.646 personas pobres de solemnidad acogidas en diversos establecimientos. Estas cifras sumadas nos darían el $51{ }^{\prime} 06 \%$ de inscritos y menesterosos, cifra absolutamente escandalosa que había que rebajar como fuera, pero era imposible para los políticos sevillanos esta rebaja por la propia estructura salarial y legal.

\footnotetext{
${ }^{15}$ Cit. por del Moral en su obra La sociedad...., pág. 82, también las siguientes citadas en la nota 22.

${ }^{16}$ Todo ello está claramente justificado en la documentación.
} 
En una doble hoja encontramos un resumen referido al año 1.903 muy útil por la proximidad casi total y por la minuciosidad con la que está realizado por distritos y por parroquias:

Hasta el 15 de Mayo de 1903

Hasta finales de Mayo de 1903

Hasta finales de Mayo de 1904
13.552 inscritos.

992 inscritos más.

1.514 inscritos más.

Lo que hace un total de 16.158 (sic). Hay que advertir que para ésta cifra y las que vienen a continuación las sumas no son correctas, pero no varían el sentido de la pretensión, saber los inscritos, saber el número de pobres y su distribución por la geografía urbana.

Esta distribución por distritos y parroquias parece ser muy exacta porque la intervención del párroco era muy importante juntamente con la del Alcalde de Barrio; nos da una radiografía muy aproximada de la pobreza y su distribución. Antes de nada algunas consideraciones de tipo formal ${ }^{17}$ :

La distribución por parroquias y distritos sanitarios, en primer lugar, trae consigo el que una parroquia, San Roque, v.g., aparezca en varios distritos, - la de San Miguel o San Marcos, por la antigua delineación de su feligresía y por la de distrito sanitario, sobre todo en las parroquias del interior, pues ahí se acumulan, pero no en Triana que contaba con sólo dos parroquias. Obsérvese la plétora parroquial en torno a las dorsales calles de San Luis y Feria, situadas en las dos líneas menos gruesas del gráfico1. Ambas eran fundamentales y conectaban la zona agrícola de la Macarena y sus contornos agrarios con la ciudad y estaban próximas a las zonas industriales del N.E. y por tanto habitadas por familias muy humildes con afiliación importante al Padrón Municipal. La ubicación parroquial y su abundancia se explica por la utilización desde antiguo de las antiguas mezquitas convertidas en parroquias. Toda la zona era de alta conflictividad social.

En segundo lugar notar que la distribución de pobres en los distritos y su densidad, es relativa, pero hay que partir de un principio y es que en todos ellos había pobres, naturalmente. Igual que el hecho de que los ricos se concentraban en algunos distritos determinados, no significaba que no los hubiera en los distritos pobres.

Es un problema de la estructura del caserío urbano. Las zonas acomodadas tendrían una densidad menor que las pobres muy pobladas, con un muy alto índice de colmatación urbanística.

Finalmente, preguntarse por la situación de quienes se apuntaban al $\mathrm{Pa}$ drón. Hemos partido de las tres o cuatro pesetas y tres o más miembros por familia, o sea, la inmensa mayoría de los obreros sevillanos. Ya hemos indi-

${ }^{17}$ Vid. los dos gráficos del presente trabajo, págs. 16 y 17. 
cado el sueldo de un médico de la Beneficencia Municipal. Por eso podemos interrogarnos sobre cuál era el límite de la pobreza, qué diferencias había entre el pobre y el obrero, qué solidaridad se establecía entre ellos, o cómo era la relación entre los pobres de barrios acomodados con sus convecinos en cuanto a prestaciones laborales, u otro tipo de relación de imposible evaluación, como podía ser el servicio doméstico, con salarios más que miserables, recogiendo sobras de todo tipo para su casa, ropa usada, etc.

Pero, si bien es verdad que son muchas las preguntas por contestar, sí se puede partir de un hecho y es que cuatro pesetas no las ganaba casi nadie del mundo laboral y un simple obrero mucho menos, por lo que la saturación, el número elevadísimo de inscritos no responde a otra cuestión primera que a la de los bajos salarios que percibían, incapaces con ellos de sostener dignamente una familia. No obstante, a pesar de todo, como gobernaban conservadores y liberales en todos los ámbitos de España en lo económico y en lo político, siempre era para un político mucho más rentable sostener a regañadientes e intentando rebajarlo siempre, un Padrón Municipal de Beneficencia que aumentar los salarios. No se olvide este punto nunca ${ }^{18}$.

Además había una realidad que era la vigencia del Real Decreto que nadie se atrevería a suprimirlo, pero sí a recortarlo y otra realidad: la miseria y hambre incuestionable que todos los sevillanos pudientes sabían que existía ${ }^{19}$.

Pasemos ahora a analizar el Padrón más próximo a 1905 que lleva el V. ${ }^{\circ}$. ${ }^{\circ}$ del Alcalde, no sin advertir la no coincidencia de distritos (diez distritos administrativos y 13 sanitarios) y parroquias ${ }^{20}$ (treinta), no olvidemos que una misma parroquia puede tener feligreses pobres en distintos distritos y pertenecer unos a un distrito de gente acomodada y otros a otro de gente pobre. Siempre que no se altere gravemente el resultado, los vamos a sumar, como puede ser el caso de San Marcos, pero es más problemático el de San Miguel, por ejemplo. Sobre las sumas inexactas, a veces, vamos a utilizar las que da fe el Sr. Sánchez Pizjuán, Secretario Municipal.

De todas las parroquias de las que da fe, han desaparecido, hasta sus cimientos, las de San Miguel en pleno centro urbano y la de Santa Lucía al noreste de la ciudad (la portada se conserva en la actual de Santa Catalina); otra ha desaparecido como parroquia, como la de Santiago.

${ }^{18}$ Sobre los salarios y la relación precios salarios, son imprescindibles las series más antiguas de Romeu Tuñón y las más modernas de González y Arenas.

${ }^{19}$ Las alusiones a estos problemas eran continuas en la prensa local y la elaboración de presupuestos familiares de todo tipo muy frecuentes. Vid. el libro citado de González, págs. 61-67.

${ }^{20}$ Es utilísima la Guía de Sevilla que Vicente GómEZ ZaRZUELA publica anualmente de una riqueza de datos realmente extraordinaria. Manejamos la de 1904 preferentemente. Estas guias se publicaron desde 1862, fundadas por D.Manuel Gómez Zarzuela, luego continuadas por Vicente Gómez Zarzuela y llegan hasta la década de los cincuenta del siglo pasado. 
Se advierte en el gráfico 2 que el menor número de parroquias se ubican al oeste de la línea ideal que fuera desde la Puerta de Jerez, por delante de la Catedral y su Parroquia del Sagrario, hasta la Alameda de Hércules, esto es, de sur a norte, sólo se encuentran cuatro / cinco parroquias. Las demás, salvo las trianeras, y San Bernardo, ésta fuera de las Rondas históricas que marcan el perfil de los dos gráficos, se encuentran situadas en la zona este, produciendo así una auténtica inflación eclesial; también es verdad que es la zona más poblada de la ciudad, pero no la más propicia al culto religioso aunque sí a nuestro benéfico Padrón.

Cabe recordar que aquí se recogen sólo parroquias oficiales, pero había, de todos es sabido, cantidad de edificios dedicados al culto que a su vez ejercían algún tipo de labores de caridad, apostolado, etc., pero no aparecen oficialmente en lo referente a nuestro trabajo.

Si trazamos la línea Norte-Sur que fuera desde la Puerta de la Carne hasta el Monasterio de San Clemente en la Barqueta, nos daría una distribución bastante exacta de los barrios humildes y pobres al NE y los acomodados al SO. Esta línea imaginaria es muy provechosa y sirve también a Arenas, de donde la tomamos, para marcar la situación de los distritos donde hay más tuberculosis, obviamente en los distritos obreros muy colmatados.

Los distritos y parroquias del NE serían las de mayor número de inscritos en el Padrón frente a los del SO. Para su mejor comprensión ofrecemos dos planos de la ciudad con los distritos administrativos y la situación de las parroquias numeradas y la línea ideal que propone el profesor Arenas, junto a las otras dos.

Para evaluar la incidencia de la tuberculosis en Sevilla, el mismo Arenas que lo toma del Dr. Ariza en el año 1.922, hace una clasificación de muertes por los distritos «administrativos» de Sevilla ${ }^{21}$. De este modo:

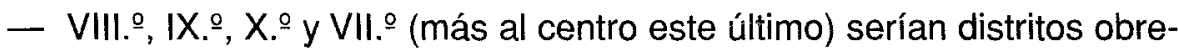
ros, pobres y por tanto con grandes inscripciones en el Padrón.

- III.. IV.. y $V^{\circ}$ - serían de clases medias. Estaba aquí el ficticio barrio de Santa Cruz, según Ariza, con porcentaje parecido al X.․

- II.. distrito acomodado y buena parte del VI.․

En 1922, el Dr. Serrano, cifra en el $75 \%$ del total de muertes, el número de tuberculosos pobres muertos por la enfermedad.

De lo que no cabe la menor duda es de que la parte de la ciudad acomodada va alejando de sí a los pobres y sus barrios, al mundo de los obreros,

${ }^{21}$ ARENAS Posada, C., La Sevilla inerme. Un estudio sobre las condiciones de vida de las clases populares sevillanas a comienzos del S.XX (1883 - 1932), Écija, 1992, págs. 55 y 56. 
creando zonas totalmente irregulares donde los problemas, enfermedades, penurias de los mismos, serían determinantes para cualquier tipo de protesta social, que por ellos comenzaría.

No hay que perder de vista que el problema de los distritos pobres y el de la tuberculosis tenían en común que era una cuestión de proporcionalidad más que de densidad, que también lo era. El hecho de que tres de cada cuarto muertos fueran pobres, aumentaría en aquellos barrios exclusivamente obreros con infraviviendas extremas y disminuiría en los acomodados en los que también habitaban pobres.

Por otra parte, el carácter nacional de la Ley y su Reglamento nos ahorra defender el carácter no localista del trabajo salvo en lo que tiene de necesario para su ubicación en un lugar y momento dado ${ }^{22}$.

En este apartado vamos a analizar, bajo diversos puntos de vista, el censo que nos ofrece el Padrón de Beneficencia ya que se nos presenta el día a día más próximo a nosotros que llega hasta el primer trimestre de 1903, aunque no recoja, salvo en los números, los 992 nuevos del segundo trimestre de dicho año y los 1.514 del año 1904, por lo que estamos ya en el año 1905.

Aunque en páginas anteriores ya hemos hablado de los gráficos, los describimos brevemente. En el 1, para mayor comodidad del lector, señalamos con tres trazos de distinto grosor, tres líneas sobre el eje N-S con dos variantes, la que pasa por Puerta de Jerez-Catedral-Sierpes-Alameda de Hércules que es una línea de raigambre clásica y un poco más al este la que pasa por Alcázar-Éncarnación-Feria, ambas perfectamente visibles en el plano. La otra línea que usara el Dr. Ariza y luego Arenas, va desde el Monasterio de San Clemente, donde el nuevo puente de la Barqueta, hasta la Puerta de la Carne; se trata de un trazado NE-SE, muy productivo para el análisis de los inscritos pues uniendo sanidad, mortalidad y pobreza que es todo uno y lo mismo, nos da la ubicación exacta de los necesitados.

\footnotetext{
${ }_{22}^{2}$ Por ejemplificar otro caso, el de Madrid, se puede ver en el libro de Carmen DEL MORAL, La sociedad madrileña fin de siglo y Baroja, sobre todo su capítulo III y IV y su acompañamiento gráfico. Para el desarrollo de una ciudad muy diferente a Sevilla, vid. El libro de Reig R., Blasquistas y clericales, Valencia, 1986, sobre la Valencia del 1900 , principalmente las págs. $314-349$, igualmente en las 279 y ss.
} 


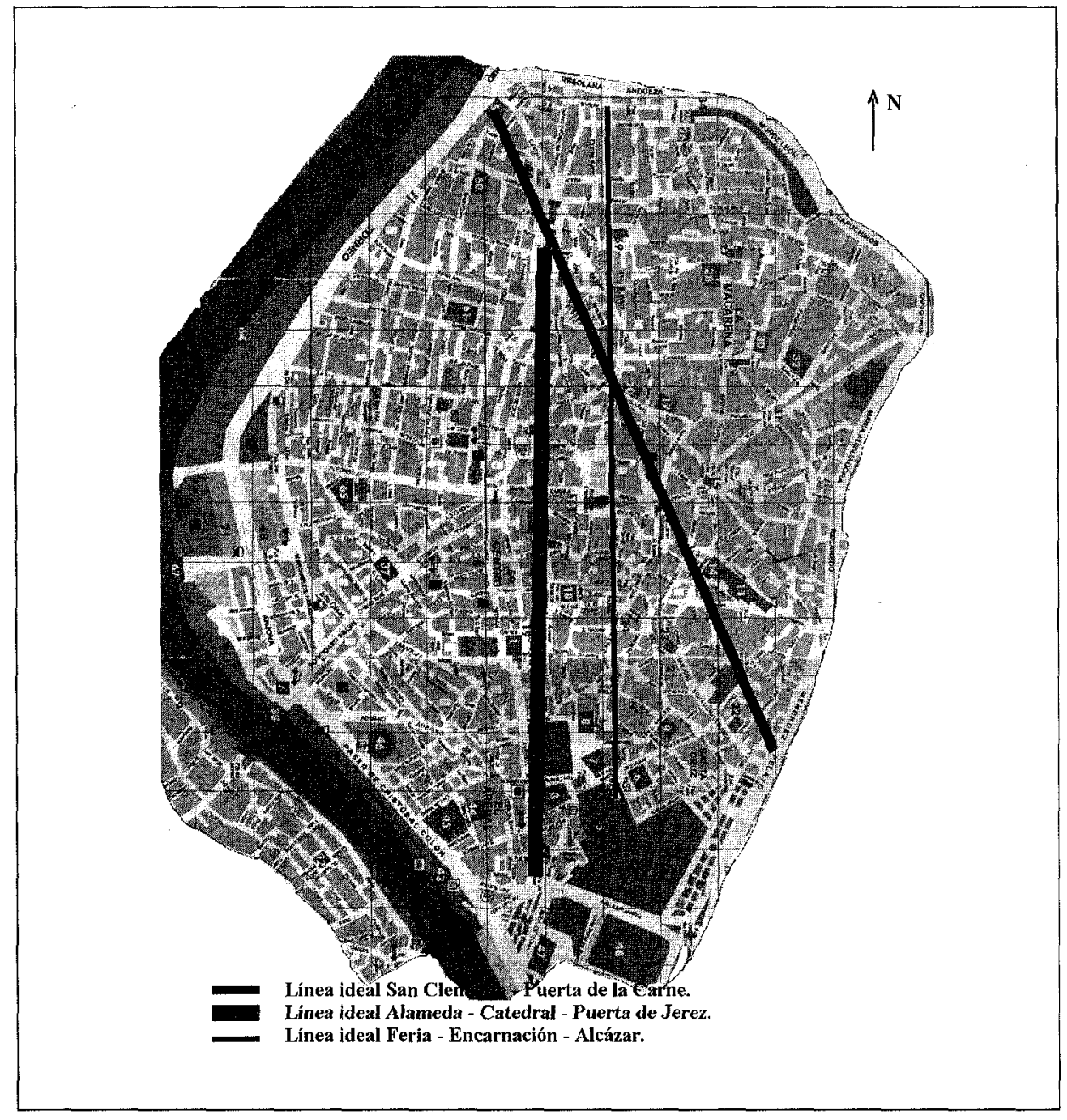

Gráfico 1.

Líneas ideales. Escala 1:20.000

Estas líneas generales se concretan más en el gráfico 2, en el que aparecen las parroquias con sus respectivos números y en trazo continuo los distritos administrativos municipales y electorales, aunque muy 'grosso modo'. Se trata de los diez distritos tradicionales, menos complicados que los que utiliza la administración municipal para la asistencia benéfica, mucho más enrevesados y de los que vamos a prescindir ya que a veces una parroquia tiene intereses benéficos en dos y hasta en tres distritos 


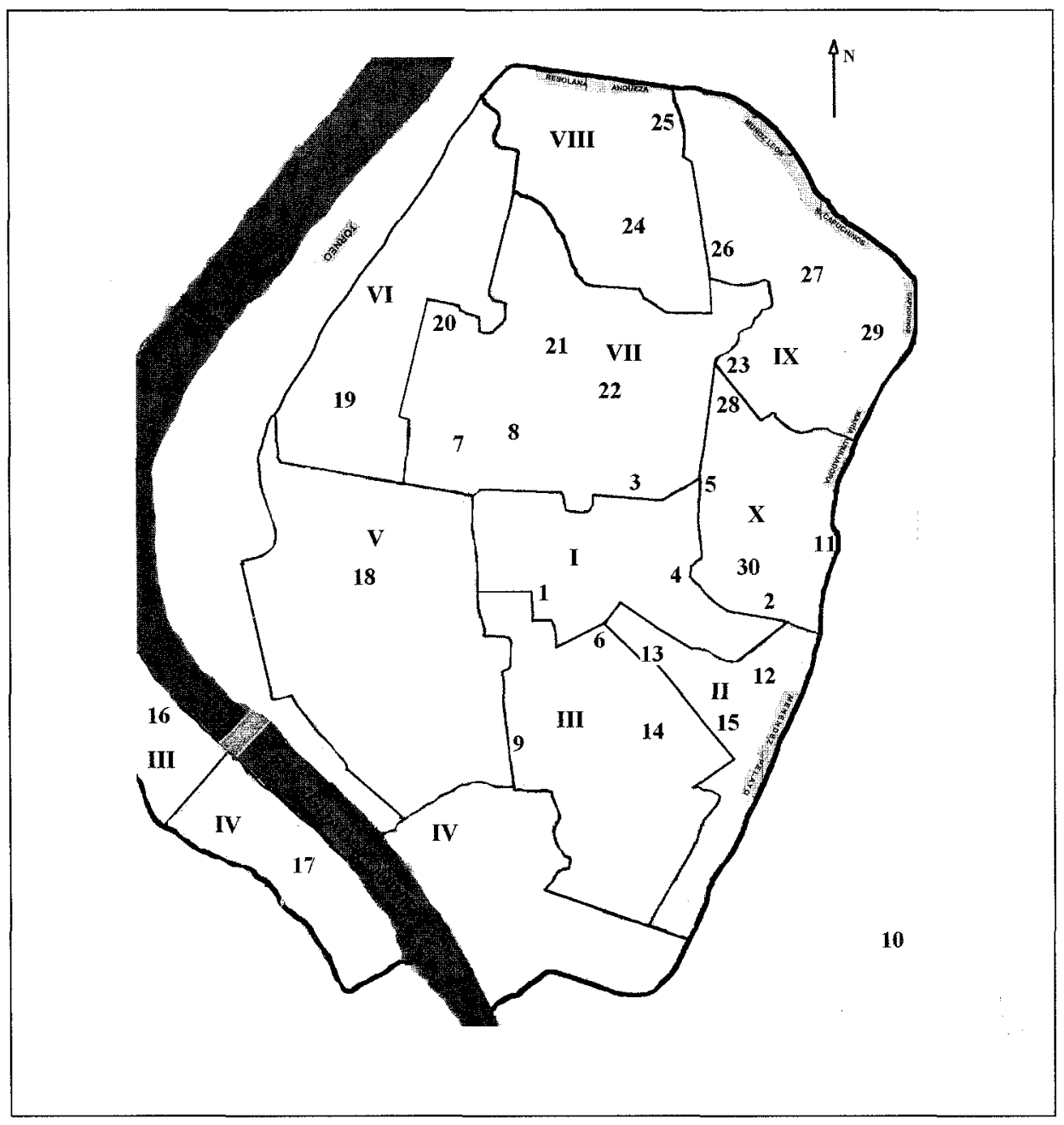

Gráfico 2.

Distritos (en romanos) y Parroquias (en árabes).

Escala 1:20.000.

Reproducimos, en la Tabla 1, las treinta parroquias sevillanas con el número de censados a la fecha indicada, primer trimestre de 1903, y antecedidas con el número con el que aparece en el gráfico 2; las hemos ordenado de mayor a menor atendiendo al número de pobres asistidos, es imprescindible para cualquier análisis. Entre paréntesis, el distrito "sanitario» al que pertenecen. Ni que decir tiene que lo mismo se podría hacer con otras ciudades españolas por lo que el localismo presente no es otra cosa que una mera incidencia: 


\begin{tabular}{|c|c|c|c|}
\hline Parroquia & N.. & N.. Familias & Distritos Sanitarios \\
\hline Santa Ana & 17 & 1.916 & V \\
\hline Omnium Sanctorum & 24 & 1.339 & $x$ \\
\hline $\mathrm{LaO}$ & 16 & 1.112 & IV \\
\hline San Roque & 11 & 723 & $\|-X\|$ \\
\hline San Bernardo & 10 & 696 & $\|$ \\
\hline San Gil & 25 & 612 & $I X-X$ \\
\hline San Lorenzo & 20 & 587 & $\mathrm{VII}$ \\
\hline Santa Catalina & 5 & 559 & XIII - VIII - I \\
\hline Sagrario & 9 & 548 & $1-V \mid$ \\
\hline Santiago & 30 & 514 & XIII \\
\hline San Román & 28 & 511 & $\mathrm{XI}$ \\
\hline Santa Marina & 28 & 477 & $I X-X$ \\
\hline San Vicente & 19 & 446 & $V|I|-V \mid$ \\
\hline San Julián & 27 & 345 & $x-V I$ \\
\hline Santa Lucía & 29 & 326 & $X I$ \\
\hline San Juan Bautista & 22 & 307 & VIII \\
\hline San Bartolomé & 12 & 265 & III \\
\hline San Marcos & 23 & 243 & $X I I I-V I I I$ \\
\hline Magdalena & 18 & 164 & $\mathrm{VI}$ \\
\hline San Esteban & 2 & 159 & $X \| 1$ \\
\hline San Nicolás & 13 & 150 & III \\
\hline Santa Cruz & 14 & 148 & III \\
\hline Santa María la Blanca & 15 & 138 & III \\
\hline Salvador & 1 & 124 & 1 \\
\hline San Martín & 21 & 123 & VIII \\
\hline San Miguel & 7 & 117 & $V I I I-I-V \mid$ \\
\hline San Pedro & 3 & 117 & VIII - I \\
\hline San IIdefonso & 4 & 74 & 1 \\
\hline San Isidoro & 6 & 44 & 1 \\
\hline San Andrés & 8 & 1 & VIII - I \\
\hline
\end{tabular}

Tabla 1.

Parroquias, número de familias inscritas y distritos sanitarios.

El Jefe de Negociado autor del borrador, declara 13.552 necesitados censados, que con el aumento del segundo trimestre y de 1904 suponen nada menos que un 18 '49\% más a añadir. Esta lista y su plasmación en el plano del gráfico 2, nos da un valor indicativo y además muy próximo de la geografía de la pobreza sevillana con dos premisas: 
- la primera, que hay pobres en todas las parroquias.

- la segunda que, se siga el método de análisis que se siga, si nos atenemos a las tres líneas del gráfico 1 , el resultado siempre es el mismo, concentración de pobreza y enfermedad hacia el norte y el este de la ciudad, con alguna variante y concentración de parroquias y pobres. La más llamativa es la línea de San Clemente-Puerta de la Carne, por ese nivel de concentración extraordinario y que luego comentaremos. Si quisiéramos distribuir por distritos municipales, tampoco erraríamos mucho si añadimos además la variante de la tuberculosis y nos darían los resultados antedichos.

Un método que sí se alejaría gravemente de los hasta ahora comentados, sería el de la Sevilla del interior y del exterior que por su excepcionalidad comentamos en primer lugar.

Cuatro parroquias existían en la Sevilla de 1904-1905 fuera de las Rondas históricas que marcan los dos gráficos: San Roque (11), que tenía también feligresía en el interior; San Bernardo (10); La O (16) y Santa Ana (17), estas dos últimas eran las parroquias trianeras. Pues bien, están entre las cinco primeras en número de censados pobres con un $32{ }^{\prime} 81 \%$ del total y con la friolera de 4.447 familias inscritas, un auténtico cáncer de la ciudad en el extrarradio y que pesan en el cómputo total en cualquier método usado. Exclusivamente en la O y en Santa Ana estaban censados 1.112 y 1.916 familias respectivamente. Eran barrios irredentos que arrastraban, además, la fama del costumbrismo y la majeza. Buena parte de ellos eran gitanos de todo oficio y otra de trabajadores de los tejares y alfares, muy numerosos, allí existentes, además de tierras de labor que absorberían la mano de obra y la mandarían al paro en los momentos de paro estructural y ahora el paro lo fue extraordinario. Se trataba, pues, de un barrio de marginalidad y de población muy alta ya que andaba en torno a los dieciocho mil habitantes lo cual significaba un $67^{\prime} 28 \%$ y un 84 ' $1 \%$ (según multipliquemos por cuatro o por cinco) de censados en el Censo general; no hay que olvidar lo prolífico de la población allí establecida. Que casi el $85 \%$ estuviera acogido al Padrón de Beneficencia nos ahorra los comentarios, pero sí podemos hacer una valoración política sobre los valores económicos y sociales de la Restauración y de las medidas adoptadas. Más difícil es explicar e imaginarse el 'modus vivendi' de estas personas basado en el trabajo ocasional y en una industria alfarera que tampoco acogería muchos brazos y unos salarios bajísimos para una población muy difícil de seguir estadística y oficialmente.

Si utilizamos la línea Puerta de Jerez-Catedral-Ayuntamiento-Sierpes hasta la Alameda de Hércules, o sea, una N-S, para toda la zona oeste de la ciudad, sólo existen las parroquias de Sagrario, Magdalena, San Vicente, San Miguel, San Lorenzo y San Andrés con 1.863 censados, el $1374 \%$ del total, para toda la zona oeste de la ciudad (no contamos las dos parroquias 
trianeras), cifra muy menguada para tan gran extensión física, pero donde vivía lo más granado y acomodado de la sociedad sevillana. En la zona este estaban las 18 parroquias restantes, más las de Triana que estaban situadas al oeste, suponían un $86^{\prime 2} 25 \%$ del total de los censados en el Padrón, aunque a veces se interfieren calles y números de las mismas o aceras, lo cual nos da una idea de los habitantes de la zona este y nos proporciona con claridad las diferencias que había entre zonas pobres y zonas acomodadas. En unas estaban las fábricas, los hospitales, el hacinamiento, la tuberculosis, las parroquias más pobres y las de mayor mortalidad: San Juan, San Pedro, San Gil, San Julián, Santa María la Blanca, San Marcos, San Román, etc., etc. Las parroquias menos mortíferas eran las del Sagrario, San Miguel, Magdalena, San Vicente, El Salvador, San Isidoro, todas al oeste de la ciudad o muy próximas con zonas de relativo alcantarillado que ya Hauser reflejó en sus planos de $1882^{23}$ y poco más se había hecho en veinte años.

Podemos tomar una línea más hacia el este, pero recta casi perfecta N/S, que nos llevaría hasta San Gil y la calle San Luis hasta el Sur y los Reales Alcázares, resulta prácticamente lo mismo que la anterior más algunas zonas nuevas correspondientes a S. Martín, El Salvador, San Juan Bautista (hoy San Juan de la Palma), que haría aumentar algo el porcentaje de la anterior, pero no de manera especialmente grave. Esto nos indica que cuanto más al este, más concentración de pobreza y enfermedad. Es decir, los barrios pobres.

Si analizamos la línea del Dr. Ariza y de Arenas para la tuberculosis que parte del Monasterio de San Clemente (la Barqueta) y llega a la popular Puerta de la Carne, nos encontramos con igual perspectiva, pero, al haber disminuido el espacio, al ser la línea algo ablicua, suben los porcentajes porque el hacinamiento aumenta vertiginosamente hacia el E.y NE de la ciudad. Todas las parroquias aquí contempladas, todas, están en zonas extremadamente conflictivas y dentro de las zonas más mortíferas de SeviIla. Y así nos encontramos con un selecto grupo de parroquias que tienen y encierran, 'velis nolis', todos los males y problemas sin que encierren bienes algunos, al contrario, conflictividades sociales, miseria, hacinamiento, muertes por tuberculosis, analfabetismo y todos los etcéteras imaginables con unas vías rectoras de la actividad de los barrios y de la vida local como la Alameda de Hércules, Calle Feria, San Luis, Sol, Santiago, Enladrillada, Plaza de San Román, Plaza de San Marcos, Puerta Osario etc. que todavía están en el recuerdo de los más ancianos y, naturalmente, en los libros. Recordemos con Arenas que el $90 \%$ de los casos de tuberculosis se producían en estos barrios y el $3 \%$ nada más, en los barrios acomodados y el $7 \%$ restante en los barrios de la periferia ${ }^{24}$.

\footnotetext{
${ }^{23}$ HAUSER, Ph., Estudios médicos topográficos, Sevilla, 1882. Especialmente los planos contenidos. Véase la nota 10 .

${ }^{24}$ ARENAS, op. cit., pág. 97.
} 
Como ya hemos transcrito el número de censados en el Padrón de Beneficencia al principio de esta segunda parte, podemos sumar las parroquias que pertenecen a esta línea oblicua y da un total de 7.405 anotados que suponen 29.620 (multiplicando por cuatro) y 37.025 (multiplicando por cinco ), un total del $54^{\prime} 64 \%$ del total del Censo en una zona, la de trazo más grueso ya que ahí se concentra tuberculosis y pobreza, que ocupa poco espacio como se puede comprobar en el gráfico 1. Este espacio soportaba, además, una colmatación altísima, las industrias de la más diversa índole: metalúrgica, corchotaponera, textil, etc., mientras que las grandes fábricas estaban fuera de las Rondas pero próximas a ellas.

Como se puede ver había zonas absolutamente irredentas a un paseo mínimo de otras, como San Andrés, de clase acomodada, que sólo tenía censado un pobre. Era la cara amable al oeste de la ciudad.

Las parroquias de la dorsal San Luis y Feria, eran auténticas representaciones de la pobreza, pero también San Julián, San Gil (la parroquia macarena) etc. No es de extrañar que cualquier tipo de manifestaciones comenzaran en estos barrios. Y las represiones también.

Algunos distritos «sanitarios» eran terribles. Veámoslos y podremos comprender, por su situación, que todos estaban en la zona más pobre y conflictiva de la ciudad:

Distrito IX. Omnium Sanctorum (24), San Gil (25) y Santa Marina (26)

Distrito X. Santa Marina (26), San Julián (27) y San Gil (25) .

Distrito XI. San Román (28) y Santa Lucía (29).

Distrito XIII. Santiago (30), Santa Catalina (5) y San Marcos (23), por no nombrar los de Triana el IV y el V.

Todos en conjunto sumaban 7.788 familias del Padrón, el 57’46\% del total. Realmente era para alarmar a los consistoriales sevillanos, pero no por los efectos, sino por las causas, aunque nadie en ningún momento se pregunte por ellas. Y así podríamos seguir multiplicando ejemplos con diversos métodos, pero siempre nos saldrá la línea N / S que origina las zonas este-oeste que vertebran la ciudad, de tal modo que el oeste va blindando su territorio ante la pobreza y las enfermedades y empujando a la mayor parte de la población hacia un verdadero submundo. Es difícil comprender a los escritores sevillanos cuando nos hablan de la ciudad de la gracia, la alegría, la despreocupación, etc. con lo que se iba cimentando una falacia histórica de una falsa Sevilla que sería alegre y hermosa sólo para unos pocos, bien es verdad ${ }^{25}$.

\footnotetext{
${ }^{25}$ Nos referimos a obras como La ciudad de la Gracia, de J. M. IzQUiERdo, Sevilla, 2001 y como las representadas en Obras de Don Juan Francisco Muñoz y Pabón, Hinojos, Huelva, 1991., del ínclito canónigo de la Catedral hispalense.
} 
Podemos deducir con to expuesto algunas conclusiones, que con el tiempo transcurrido, parecen de pura lógica, pero que en su momento tenía un dramatismo extraordinario.

En el fondo de todo esto latía el sentimiento de fracaso social de la Restauración que necesariamente buscaría en los procesos de protesta y en los revolucionarios su válvula de escape que era cerrada férreamente en el momento preciso. Salvo medidas legales que se quedaban en el papel en muchos casos y subidas salariales que no llegaban puesto que hasta 1917 hubo un estancamiento notable y un aumento de precios constante, poca mejora se observa en la sociedad menos favorecida del primer tercio del siglo y como comprobante tenemos el testigo molesto de la tuberculosis imparable en su aumento. Ya que todavía en 1925, la mortalidad producida por la enfermedad, era el doble de la media española. La creación de los dispensarios antituberculosos respondían a los efectos pero no a las causas de la enfermedad que en su origen permanecían intactas.

Dice Arenas Posadas que «El problema de la pobreza en la Sevilla del comienzo del siglo XX, como en las ciudades europeas de dos o tres siglos antes, no derivaba del número más o menos crecido de «ociosos en edad de trabajar», sino del hecho de que los conceptos paro - trabajo - miseria enfermedad eran simultáneos para un importante colectivo de trabajadores ${ }^{26}$. Con esta perspectiva era imposible tener otro Padrón que el que había, el cual, a pesar de las revisiones, al poco tiempo quedaba estrecho y había que volver a la revisión ya que las estructuras salariales y sociales eran las mismas. Pensemos que desde finales de siglo y hasta 1917, los salarios estuvieron estancados. Por eso las conclusiones de este trabajo no pueden olvidar este principio.

1. La reducción del Padrón se debe a motivos extrasociales: una visión deformada de la Sevilla de la época, una idea equivocada de la pobreza, un concepto puramente de economía municipal y una idea falsa del prestigio de la ciudad que sólo estaba en las mentes de la clase acomodada que vivía de espaldas ante una realidad, más que conocida, de la que se aprovechaba, como nos informa el Censo de 1900 que registra un 13'4 de la población activa con pagos miserables menores aún que los de cualquier peón; se trata de una fuerza laboral formada por 6.746 censados, el $40 \%$ del sector servicios, cifra algo superior a los asalariados del comercio.

2. Como ocurre siempre, el binomio proletariado (industrial o agrícola) y pobreza va unido al problema de la vivienda y su ubicación como comprobamos en este trabajo. Hay que añadir, naturalmente, como corolario, la enfermedad.

${ }^{26}$ Arenas Posadas, C., Sevilla y el Estado 1892 - 1923. Una Perspectiva Local de la Formación del Capitalismo en España, Sevilla, 1995, pág. 194. 
El padrón municipal de beneficiencia y la geografía de la pobreza oficial sevillana c. 1905.

3. Tenidos durante mucho tiempo los 'corrales' como verdaderas viviendas absolutamente insanas, eran superados por las piezas inhumanas nacidas de continuas divisiones de antiguas casonas. Sabemos que había viviendas de hasta cinco metros cuadrados. La enfermedad y la pobreza anidaban en ellos.

4. La línea N/S marca un sentido de desarrollo, la zona noble al Sur. Pero la marca de desarrollo/subdesarrollo social está en la $E / O$, mostrando dos ciudades diferentes: la acomodada (O) y la pobre (E). Naturalmente, esto es lo más significativo para este trabajo.

5. Cabe constatar la existencia de zonas completamente malditas que necesariamente debían llamar la atención; por eso el número de acogidos al Padrón no puede extrañarnos, de ninguna manera, por muy elevado que sea, precisamente porque conocemos los salarios y en 1905 el paro necesariamente muy alto por la crisis agrícola.

Para comprender bien la realidad social de la ciudad, a los acogidos al Padrón, hay que añadir 13.644 pobres de solemnidad de financiación diferente. Cabe preguntarse si estas cifras podían ser toleradas por cualquier núcleo urbano. La respuesta es obvia y los responsables también.

Ya hemos hablado de los médicos y farmacéuticos pero quienes libraban el apuntamiento para el Padrón eran los Alcaldes de Barrio y los Párrocos a los que se acusará de blandura. No cabe duda de que, junto con los médicos, eran los mejores conocedores de la situación social.

Como podemos constatar los pobres no se distribuían equitativamente por barrios: los pobres vivían y estaban siempre en los mismos barrios. 